\title{
Proactive regulation of axial crushing behavior of thin-walled circular tube by gradient grooves
}

\author{
Yanpeng Wei ${ }^{\mathrm{a}, *}$, Zhe Yang ${ }^{\mathrm{a}}$, He Yan ${ }^{\mathrm{b}}$, Yacong Guo ${ }^{\mathrm{a}}$, Xianqian $\mathrm{Wu}^{\mathrm{a}}$, Chenguang Huang ${ }^{\mathrm{a}}$ \\ a Key Laboratory for Mechanics in Fluid Solid Coupling Systems, Institute of Mechanics, Chinese Academy of Sciences, Beijing 100190, China \\ ${ }^{\mathrm{b}}$ Institute of Nuclear and New Energy Technology of Tsinghua University, Collaborative Innovation Center for Advanced Nuclear Energy Technology, \\ Beijing 100084, China
}

\section{A R T I C L E I N F O}

Article history:

Received 25 October 2015

Received in revised form

19 January 2016

Accepted 28 January 2016

Available online 3 February 2016

Keywords:

Gradient grooved tube (GGT)

Proactive regulation

Progressive buckling

Half wavelength

Sectionalized crushing force

\begin{abstract}
A B S T R A C T
The progressive buckling behavior of a gradient grooved tube (GGT) designed for high temperature gas cooled reactor (HTR) is systematically studied in this paper. Based on the plastic hinge formation process, a sectionalized theoretical model is established to predict the quadratic upward trend of crushing force of GGT. The ordered and stable energy absorbing process of GGT is examined by low-speed axial impact using drop hammer test machine. The effect of proactive regulation parameters, including nondimensional groove width $\mathrm{W} /\left(\mathrm{h}_{0}+h\right)$, groove depth $h_{0} / h$ and half wavelength $(H+W-h) / \sqrt{D h}$ on buckling modes and force-displacement curve is determined by experiments and FE simulation. The non-dimensional groove width $W /\left(h+h_{0}\right)$ is determined as $\pi / 2$ according to the geometry coordination of bending grooves. The non-dimensional groove depth $h_{0} / h$ influences local buckling behavior and the critical value 0.6 for regular bending has been determined by numerical simulation. The quadratic trend force-displacement curves of GGT can be actively controlled by $(H+W-h) / \sqrt{D h}$ within a certain range less than 1.2. Free-fall impact experiments show that GGT has a better stability than perfect tube when used as an energy absorber to protect the graphite in HTR from slender control rod impact.
\end{abstract}

(c) 2016 Elsevier Ltd. All rights reserved.

\section{Introduction}

Energy absorption is a critical issue in the protection of structures from impact and collision damage. Thin-walled metal tube is one of the most commonly used energy absorber as the simple structure and efficient energy absorbing capacity. There are a lot of researches in this area over the past decades and the review articles and books outlined the main findings [1-4].

According to different loading and boundary conditions, thinwalled tubes can dissipate the impact energy through different deformation modes. The modes for axial loading conditions mainly include axial crushing [5-8], tube inversion [9-12] and tube splitting [13-15]. Axial crushing of the tube is one of the most efficient and reliable modes due to the high specific energy absorption, high stroke and volumetric efficiency and progressive buckling behavior. Tube inversion and tube splitting often need a shaped die or a clamp to initiate and retain the process, these modes have a relatively flatten force-displacement curves but have lower adaptability to severe external conditions such as oblique impact than axial crushing mode. The modes for lateral

\footnotetext{
* Corresponding author. Tel.: +8610 82544256.

E-mail address: weiyanpeng@imech.ac.cn (Y. Wei).
}

loading conditions include lateral compression [16-21] and lateral indentation [21-24]. The lateral modes have the advantage to use in narrow energy-absorbing space because of the low compressing displacement and these modes have smooth force-deflection responses though the energy absorbing efficiency is lower than the axial crushing mode.

Thin-walled metal tube may be the best energy absorber that can be used in graphite core of high temperature gas cooled reactor (HTR) due to the narrow control rod channel space, high temperature environment and super clean demands [25-27]. For a perfect tube, the oscillation of crushing force and the erratic buckling mode are natural. In axial impact event, the critical buckling force (the peak crushing force) is much higher than the average one, and the following peak forces have a decreasing trend due to strain rate effect. Meanwhile, the buckling mode is sensitive to the diameter-thickness ratio $D / h[28,29]$. In a wide range of $D / h$, a tube starts in concertina mode but transforms into diamond mode during buckling [5]. Moreover, the plastic hinges tend to start at the distal end due to the stress wave reflection. All these factors ease to lead lateral overturn when the absorber is impacted by a slender free-fall control rod with no lateral restriction. So the crushing force and buckling mode must be finely regulated to form a stable collapse process. 


\begin{tabular}{|c|c|c|c|}
\hline \multicolumn{2}{|c|}{ Nomenclature } & $E_{\text {ring }}$ & stretching and compressing energy of ring \\
\hline$D$ & diameter of tube & $m_{f}$ & eccentric factor (ratio of outward fold length to total \\
\hline$h$ & wall thickness of tube & & fold length) \\
\hline$L$ & length of tube & $Y$ & yield stress of tube material \\
\hline$m$ & location number of groove & $Y^{\prime}$ & yield stress in a state of plane strain using Von-Mises \\
\hline$h_{m}$ & depth of $m$ th groove & & yield condition \\
\hline$h_{0}$ & initial depth of groove & $V$ & impact velocity \\
\hline$t$ & variation of groove depth & $\rho$ & density of material \\
\hline$W$ & ring length between grooves & $E$ & Young's modulus \\
\hline$H$ & width of grooves & $\mu$ & Poisson's ratio \\
\hline$n$ & fold number & $h_{\text {last }}$ & last groove depth \\
\hline$F_{n}$ & average crushing force of $n$th fold & $h_{\text {mid }}$ & non-dimensional middle groove wall thickness of \\
\hline$E_{f n}$ & energy absorbed by $n$th fold & & $n$th fold \\
\hline$S_{n}$ & crushing displacement of $n$th fold & $F_{n}^{\text {impact }}$ & $\begin{array}{l}\text { average crushing force of } n \text {th fold under impact } \\
\text { conditions }\end{array}$ \\
\hline $\begin{array}{l}E_{f} \\
E_{h}\end{array}$ & bending energy of plastic hinge & $F_{n}^{\text {static }}$ & average crushing force of $n$th fold under quasi-static \\
\hline$E_{S C}$ & stretching and compressing energy of groove & & conditions \\
\hline$E_{c}$ & compressing energy of groove & $\alpha$ & hardening parameter \\
\hline$E_{s}$ & stretching energy of groove & & \\
\hline
\end{tabular}

Initial imperfections such as local dents, holes, grooves, ribs and pre-buckle on the tube are commonly used to regulate the collapse process. In summary, the effect of initial imperfections can (a) initiate a specific axial collapse mode, (b) stabilize the collapse process, and (c) for axial crush response, reduce the peak load or optimize specific crush characteristics [30,31]. The initial imperfections can be extensively applied to the entire tube, therefore the buckling mode can be controlled to some extent. Adachi et al. analyzed the effects of stiff ribs on the improvement of energy absorption characteristics [32] and found the ribs can be used to improve or adjust energy absorption characteristics, and a critical distance between ribs for mode conversion was determined by experimental methods. Hosseinipour and Daneshi $[33,34]$ performed quasi-static crushing tests for the tubes with circumferential grooves alternately placed inside and outside the tubes at predetermined intervals. They found uniform annular grooves can control the folding location, reduce the peak load and make the load pulse evenly. Theoretical analysis has been made for the mean crushing force and met a good agreement with the experimental results. a

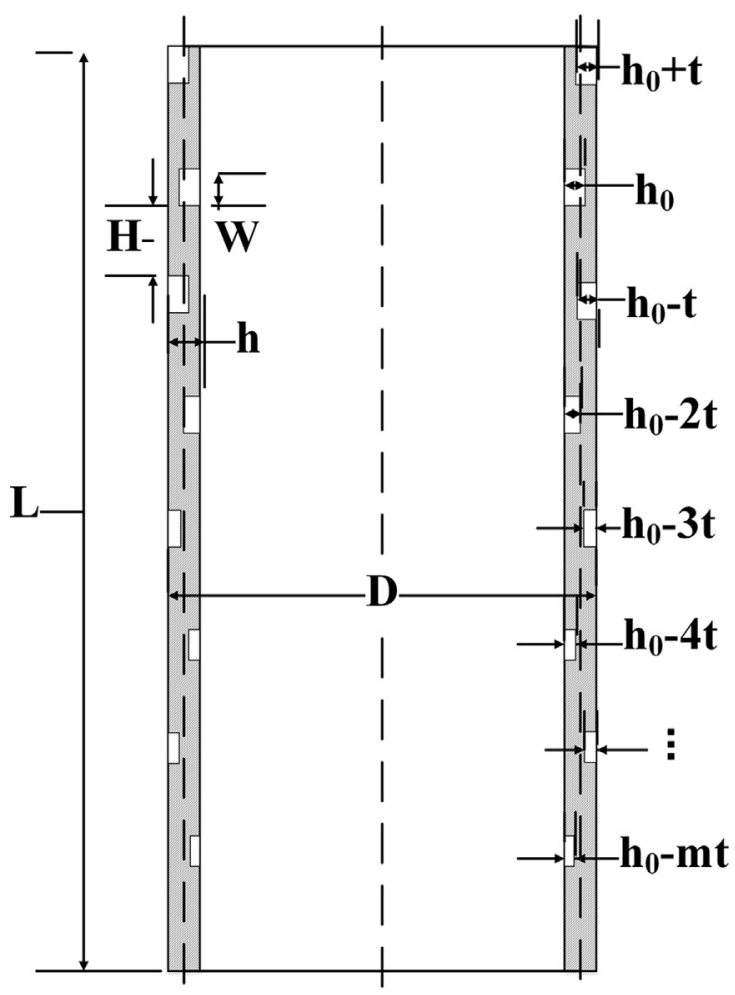

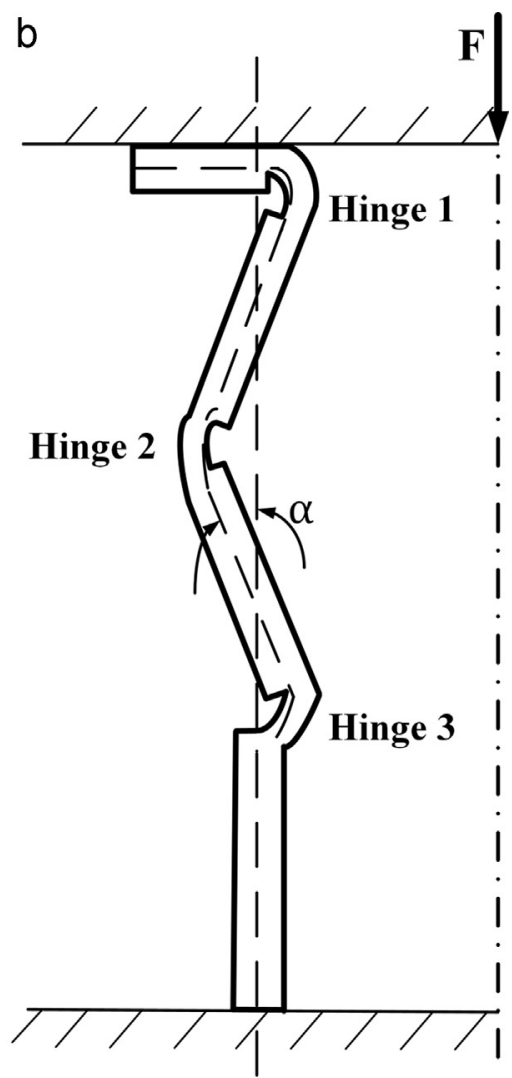

Fig. 1. (a) Schematic diagram of the arrangement of circumferential rectangular grooves with linear gradient depths; (b) a fold formation model of GGT. 
However, whether grooves or ribs, they have the same geometry and uniform positional distribution along the tube, which will cause uncertainty of the buckling sequence. In conventional energy-absorbing components, frusta is a kind of gradient structure which can export an upward-trending crushing force leading to a stable buckling sequence [35]. Tapered circular tubes with graded wall thickness show similar upward trends with frusta and have a larger design domain than the straight tube [36]. Although the geometry of frusta and tapered tubes bring manufacturing difficulties and application limitations in narrow control rod channels, they still present important characteristics that a stable energy-absorbing structure should have: consistent buckling mode, stable buckling sequence, small oscillations and upward trend of crushing force.

In this work, the gradient grooves are arranged along the thinwalled circular tube to regulate energy-absorbing process. A sectionalized theoretical model is proposed to predict the crushing force during the buckling process. The influence of several nondimensional controllable geometric parameters - including initial groove depth $\left(h_{0} / h\right)$, groove width $\left(\mathrm{W} /\left(\mathrm{h}_{0}+h\right)\right)$ and half wavelength $((H+W-h) / \sqrt{D h})$ on the buckling modes and trend of crushing force - are studied by systematic numerical simulations and experiments. Free-fall impact experiments demonstrated that gradient grooved tube (GGT) has a better stability than perfect tube when used as an absorber to protect the graphite in HTR from slender control rod.

\section{Theoretical analysis}

The crushing process is proactively regulated by a series of grooves presetting along the stainless steel tube. As shown in Fig. 1a, circumferential rectangular grooves have a linear gradient distribution $\left(h_{m}=h_{0}-(m-2) t\right)$, where $h_{0}$ is the initial depth at the top end (the second groove from the top end, the first one acts as a trigger and its depth is $\left.h_{0}+t\right), m$ is the groove location number and $t$ is the depth variation. $W$ is the width of a single groove, and $H$ is the width of the space between adjacent grooves.

A theoretical crushing model for GGT under axial loading is shown in Fig. 1b. Within the range of reasonable groove parameters, plastic hinges will be limited to the circumferential grooves, and as such, the tubes would buckle in perfect concertina mode. The average crushing force of the $n$th fold $\left(F_{n}\right)$ is the ratio of the absorbed energy $\left(E_{f n}\right)$ and the crushing displacement $\left(S_{n}\right)$.

$F_{n}=\frac{E_{f n}}{S_{n}}$

$S_{n}$ is a distance of two times the ring width subtracting the residual length $2 h$, which are the same for each fold:

$S_{n}=2(H+W-h)$

This model here is based on the assumption of Alexander's model [37], the tube is made of a rigid, perfect plastic material and the work of loading $\left(E_{f}\right)$ in an integral fold is converted into the plastic bending energy $\left(E_{b}\right)$, the stretching and compressing energy $\left(E_{s c}\right)$ and the plastic work of the rings between grooves $\left(E_{\text {ring }}\right)$. And these three parts have no interaction during the process. The eccentric factor $m_{f}$ (ratio of outward fold length to total fold length) shown in Singace's work is about 0.5-0.7 [38,39], for the simplicity of the model and convenience of calculation, $m_{f}$ is assumed to be 0.5 in this model.

For the $n$th fold, the energy absorption $\left(E_{f n}\right)$ can be expressed as Eq. (3):

$$
\begin{aligned}
E_{f n}= & E_{b}^{n}+E_{s c}^{n}+E_{r i n g}^{n}=E_{b(2 n-1)}+E_{b(2 n)}+E_{b(2 n+1)}+E_{c(2 n-1)} \\
& +E_{s(2 n)}+E_{c(2 n+1)}+E_{\text {ring }}
\end{aligned}
$$

where $E_{b(2 n-1)}, E_{b(2 n)}$, and $E_{b(2 n+1)}$ are the bending energy of the plastic hinges located on the $(2 n-1)$ th, $2 n$th and $(2 n+1)$ th grooves respectively. $E_{c(2 n-1)}$ and $E_{c(2 n+1)}$ are the compressing energy of the $(2 n-1)$ th and $(2 n+1)$ th grooves, which go inside the midline of the tube wall and $E_{s(2 n)}$ is the stretching energy of the $2 n$th groove which goes outside the midline of the tube wall. $E_{\text {ring }}$ signifies the energy absorbed by the two rings between the grooves.

The bending energy of the $(2 n-1)$ th, $(2 n)$ th and $(2 n+1)$ th grooves can be expressed as Eqs. (4)-(6):

$$
\begin{aligned}
E_{b(2 n-1)}= & M_{p(2 n-1)} \int_{0}^{\pi / 2} \pi(D-(H+W) \sin \alpha) d \alpha \\
= & \frac{\pi^{2}}{2} D M_{p(2 n-1)}-\pi M_{p(2 n-1)}(H+W) \\
E_{b(2 n)}= & 2 M_{p(2 n)} \int_{0}^{\pi / 2} \pi(D+(H+W) \sin \alpha) d \alpha \\
= & \pi^{2} D M_{p(2 n)}+2 \pi M_{p(2 n)}(H+W) \\
E_{b(2 n+1)}= & M_{p(2 n+1)} \int_{0}^{\pi / 2} \pi(D-(H+W) \sin \alpha) d \alpha \\
= & \frac{\pi^{2}}{2} D M_{p(2 n+1)}-\pi M_{p(2 n+1)}(H+W)
\end{aligned}
$$

$M_{p}$ is the plastic bending limit moment using von Mises' yield condition and assuming the tube is in a state of plane strain for which $Y^{\prime}=2 Y / \sqrt{3}$. The plastic bending moment for the $m$ th groove is expressed as follows:

$M_{p(m)}=\left(\frac{2}{\sqrt{3}} Y\right) \frac{\left(h-h_{0}+(m-2) t\right)^{2}}{4}$

The plastic bending energy of the three grooves for the $n$th fold is then:

$$
\begin{aligned}
E_{b}^{n}= & E_{b(2 n-1)}+E_{b(2 n)}+E_{b(2 n+1)}=\frac{\pi^{2}}{2} D\left(M_{p(2 n-1)}+2 M_{p(2 n)}\right. \\
& \left.+M_{p(2 n+1)}\right)-\pi(H+W)\left(-M_{p(2 n-1)}+2 M_{p(2 n)}-M_{p(2 n+1)}\right) \\
= & \frac{\pi^{2} Y D\left(h-h_{0}+2 n t-2 t\right)^{2}}{\sqrt{3}}+\frac{\pi^{2} Y D t^{2}}{2 \sqrt{3}}-\frac{\pi Y(H+W) t^{2}}{2 \sqrt{3}}
\end{aligned}
$$

The second and the third terms can be ignored as $t^{2}$ is a minor term compared with $\left(h-h_{0}\right)^{2}$. Thus, the bending energy of the three grooves for the $n$th fold is approximately equal to:

$E_{b}^{n} \approx \frac{\pi^{2} Y D\left(h-h_{0}+2 n t-2 t\right)^{2}}{\sqrt{3}}$

From Eq. (9), the bending energy of the three grooves can only be affected by the middle groove wall thickness $\left(h-h_{0}+2 n t-2 t\right)$.

The stretching and compressing energy of the $(2 n-1)$ th, $(2 n)$ th and $(2 n+1)$ th grooves due to diameter variation can be expressed as Eqs. (10)-(12) below, and the sum of them is expressed as Eq. (13):

$$
\begin{aligned}
& E_{c(2 n-1)}=\pi Y\left(h-h_{0}+2 n t-3 t\right)\left(\frac{W^{2}}{4}+\frac{H W}{2}\right) \\
& E_{s(2 n)}=2 \pi Y\left(h-h_{0}+2 n t-2 t\right)\left(\frac{W^{2}}{4}+\frac{H W}{2}\right) \\
& E_{c(2 n+1)}=\pi Y\left(h-h_{0}+2 n t-t\right)\left(\frac{W^{2}}{4}+\frac{H W}{2}\right) \\
& E_{s c}^{n}=E_{c(2 n-1)}+E_{s(2 n)}+E_{c(2 n+1)}=\pi Y\left(h-h_{0}+2 n t-2 t\right)\left(W^{2}+2 H W\right)
\end{aligned}
$$


As the groove widths and ring lengths are constants for a specific model, the energy absorbed by the two rings between the grooves is the same among the folds:

$E_{\text {ring }}^{n}=\pi Y h H^{2}$

The energy absorbed by the $n$th fold $E_{f n}$ can be expressed as:

$$
\begin{aligned}
E_{f n}=E_{b}^{n} & +E_{s c}^{n}+E_{\text {ring }}^{n}=\frac{\pi^{2} Y D\left(h-h_{0}+2 n t-2 t\right)^{2}}{\sqrt{3}}+\pi Y\left(h-h_{0}\right. \\
& +2 n t-2 t)\left(W^{2}+2 H W\right)+\pi Y h H^{2}
\end{aligned}
$$

The average crushing force of the $n$th fold is:

$$
\begin{aligned}
F_{n}= & \frac{E_{f n}}{S_{n}}=\frac{\pi^{2} Y D h^{2}}{2 \sqrt{3}(H+W-h)}\left(1-\frac{h_{0}-2 n t+2 t}{h}\right)^{2} \\
& +\frac{\pi Y D^{\frac{1}{2}} h\left(W^{2}+2 H W\right)}{2(H+W-h)}\left(1-\frac{h_{0}-2 n t+2 t}{h}\right)+\frac{\pi Y D^{\frac{1}{2}} h H^{2}}{2(H+W-h)}
\end{aligned}
$$

For a specific model, $n$ is the only independent variable in this function. The Eq. (16) above, is expressed as a quadratic polynomial, the independent variable is the non-dimensional middle groove wall thickness for the $n$th fold $\left(h_{\text {mid }}=1-\frac{h_{0}-2 n t+2 t}{h}\right)$. The quadratic term represents the bending energy contribution by the grooves. The primary term represents the stretching and compressing energy contribution by the grooves, and the constant term represents the energy contribution by the rings. For uniform grooves without depth variation $(t=0)$, and if the eccentric factor $m_{f}$ is set as 1 or 0 (totally external or internal folding) while the half wavelength $(H+W-h)$ is set as $(H+W)$, the average crushing force Eq. (16) will be consistent with the theoretical analysis in reference [34].

It is worth noting that three non-dimensional parameters $h_{0} / h$, $\mathrm{W} /\left(\mathrm{h}_{0}+h\right)$ and $(H+W-h) / \sqrt{\mathrm{Dh}}$, have significant effects on the buckling process of GGT. $h_{0} / h$ is the ratio of the depth of the grooves to the wall thickness of the tube. $W /\left(\mathrm{h}_{0}+h\right)$ is the ratio of groove width to wall thickness. $(H+W-h) / \sqrt{\mathrm{Dh}}$ is the nondimensional half wavelength: the numerator $(H+W-h)$ is the artificial half wavelength of the GGT, and the denominator $(\sqrt{D h})$ is the inherent half wavelength of a perfect tube, which is the intrinsic property of the perfect tube $[7,37,38,40]$. The concertina buckling mode and half wavelength of GGT can be proactively regulated by $(H+W-h) / \sqrt{\mathrm{Dh}}$.

\section{Experimental setup}

The impact experiment was carried out using drop hammer equipment with a $200 \mathrm{~kg}$ load mass block as shown in Fig. 2. The hammer was dropped from a height of $3.7 \mathrm{~m}$, and via gravity acceleration it reached a velocity $\mathrm{V} \approx 8.5 \mathrm{~m} / \mathrm{s}$ before impact.

The buckling process was captured by a high-speed camera with $5700 \mathrm{fps}$ at full resolution $(1024 \times 1024$ pixels $)$. A faster mode of $8000 \mathrm{fps}$ at lower resolution $(704 \times 992$ pixels) was selected to capture the detailed deformation characteristics of the buckling behavior. One $200 \mathrm{~W}$ LED $(19,000 \mathrm{~lm})$ was used as a light source to supply adequate luminous flux to the camera for such high speeds. The displacement and speed of the drop hammer could be obtained by the two-dimensional digital image correlation (2D DIC) method using the images captured by the high-speed camera. The tracing area was a rectangular speckled band on the drop hammer, as shown in Fig. 3, on a plane parallel to the CCD sensor during the buckling process. The length of the rectangle $(100 \mathrm{~mm})$ was selected as the reference to calibrate the real distance per pixel.

A pressure sensor assembly was located at the bottom to measure the transient crushing force during the buckling process. The piezoelectric coefficient of pressure sensor was $30449 \mathrm{~N} / \mathrm{V}$, measured by a material testing system. All voltage signals were obtained by a high dynamic data acquisition unit with $40 \mathrm{kHz}$ sampling frequency.

\section{Numerical model}

The finite element model has been established by commercial FE code LS-DYNA 971 to study the buckling process and local deformation of GGTs. As shown in Fig. 4, three parts are defined in this model: the impact hammer, GGT and back plate. All the parts are discretized with 8 node hexahedral elements. The tubes are modeled with 150 elements in longitudinal direction, 60 elements in circumferential direction and 6 elements through the shell thickness. The contact between GGT and back plate is "tied" while all the others are set as "automatic single surface". The friction
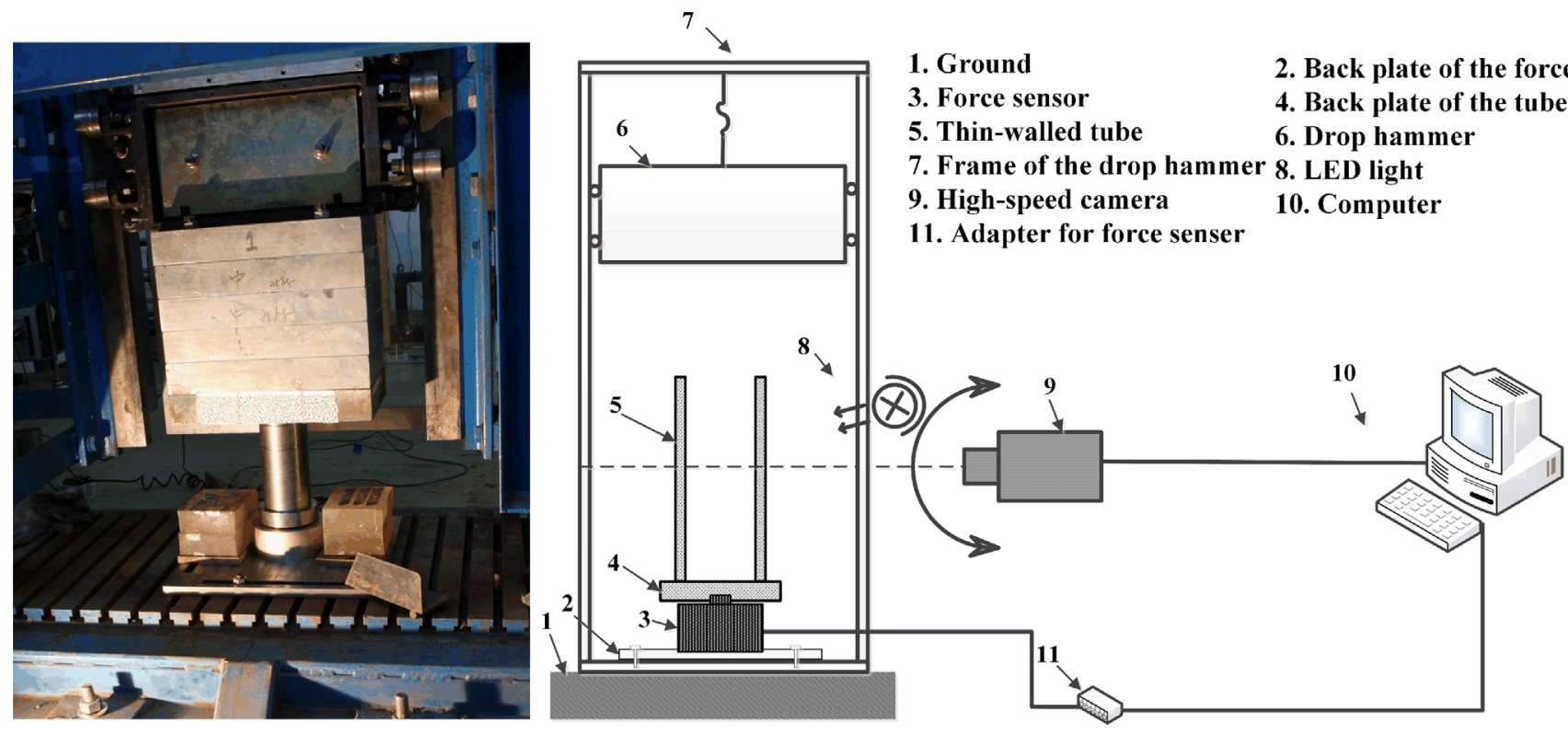

Fig. 2. Schematic diagram of the experimental setup. 

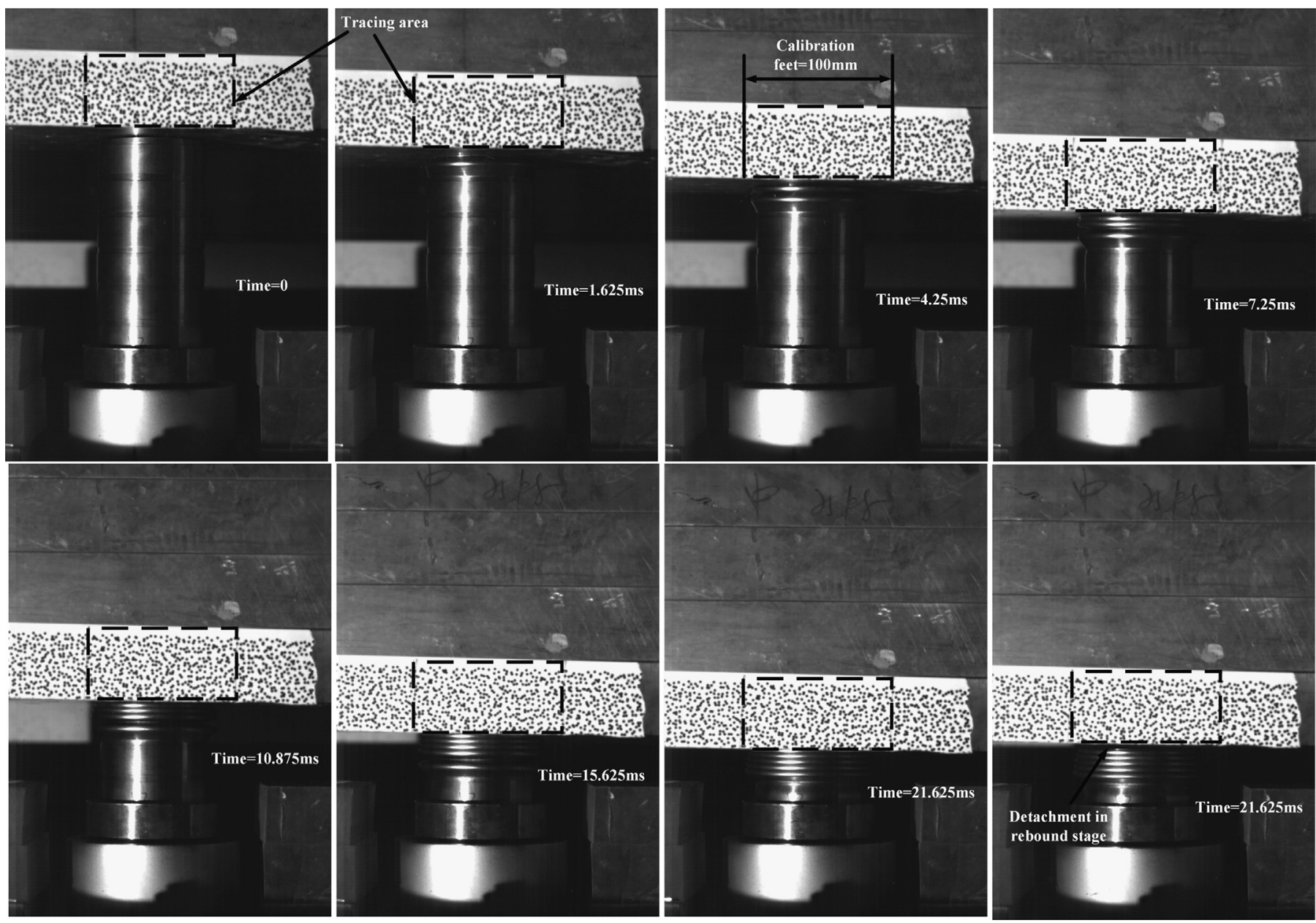

Fig. 3. High-speed images with tracing area for DIC method.

coefficient is set as 0.15 for all the contacts. The impact hammer is modeled as a rigid body, the density is set as $2.264^{*} 10^{6} \mathrm{~kg} / \mathrm{m}^{3}$ to simulate the hammer weighting $200 \mathrm{~kg}$. The back plate is modeled as an elastic body with the bottom surface fixed in all dimensions. The material parameters in the present model are listed in Table 1. All the GGTs in this study have the same geometry: $150-\mathrm{mm}-$ length, 80-mm-diameter and 2-mm-thickness.

The strain rate hardening effect and the strain hardening effect of the 304 stainless steel material are described using the Johnson-Cook constitutive model [41]. The high temperature soften effect is not considered here, then the plastic flow stress of the material is expressed as Eq. (17).

$\sigma_{e q}=\left(A+B \varepsilon^{n}\right)\left(1+C \ln \dot{\varepsilon}^{*}\right)$

where $\varepsilon$ is the equivalent plastic strain, $\dot{\varepsilon}^{*}=\dot{\varepsilon} / \dot{\varepsilon}_{0}$ is the dimensionless plastic strain rate for $\dot{\varepsilon}_{0}=1 \mathrm{~s}^{-1}$. The first bracket in Eq. (17) gives the plastic flow stress as a function of strain when strain rate $\dot{\varepsilon}^{*}=1$. The second bracket expresses the strain rate hardening effects. The material constants $A=278 \mathrm{MPa}, B=1300 \mathrm{MPa}, n=0.80$ and $C=0.072$ are obtained from quasi-static tensile tests and Hopkinson bar tests at different strain rates from reference [42].

\section{Results and discussion}

\subsection{Validity of numerical model}

There is no need to carry out large scale experiments to evaluate the effects of proactive regulation if an exact numerical model exists. In this section therefore, the validity of the numerical model is examined systematically by experimental results at $\mathrm{h}_{0} / h=0.6$, $\mathrm{W} /\left(\mathrm{h}_{0}+h\right)=\pi / 2$ and $(H+W-h) / \sqrt{\mathrm{Dh}}=1$.

As shown in Fig. 5, the GGT buckles from the proximal end where the deepest trigger groove is located, and sequentially to the distal end. Both experimental and numerical results show that all the plastic hinges are strictly limited to the preset grooves to form five complete concertina folds in this model. The local plastic hinge goes outside at thinner groove and inside at the outer groove, the same as the assumed theoretical model, together with Hosseinipour and Daneshi's model [34]. The eccentric factor $\left(m_{f}\right)$ in the numerical model is about 0.6 , which is consistent with experimental results.

Fig. 6 shows good agreement between 2D DIC and numerical results of displacement-time and velocity-time curves in the initial buckling stage and post-buckling stage. Meanwhile, there is also good consistency in the force-displacement $(F-D)$ curve from both the pressure sensor and numerical model, which will be discussed in detail in Section 5.3.1.

The numerical model can be given complete validation as it can predict the buckling modes, crushing displacement, velocity and crushing force process well. All the numerical cases discussed below have the same settings of material parameters, mesh size, loading conditions, boundary conditions and friction coefficient.

Above all, the gradient grooves on the tube can control the fold formation position (plastic hinges on the grooves) and sequence (from the deepest groove end to the other sequentially), which show the effect of proactive regulation by the gradient grooves of the buckling process. 


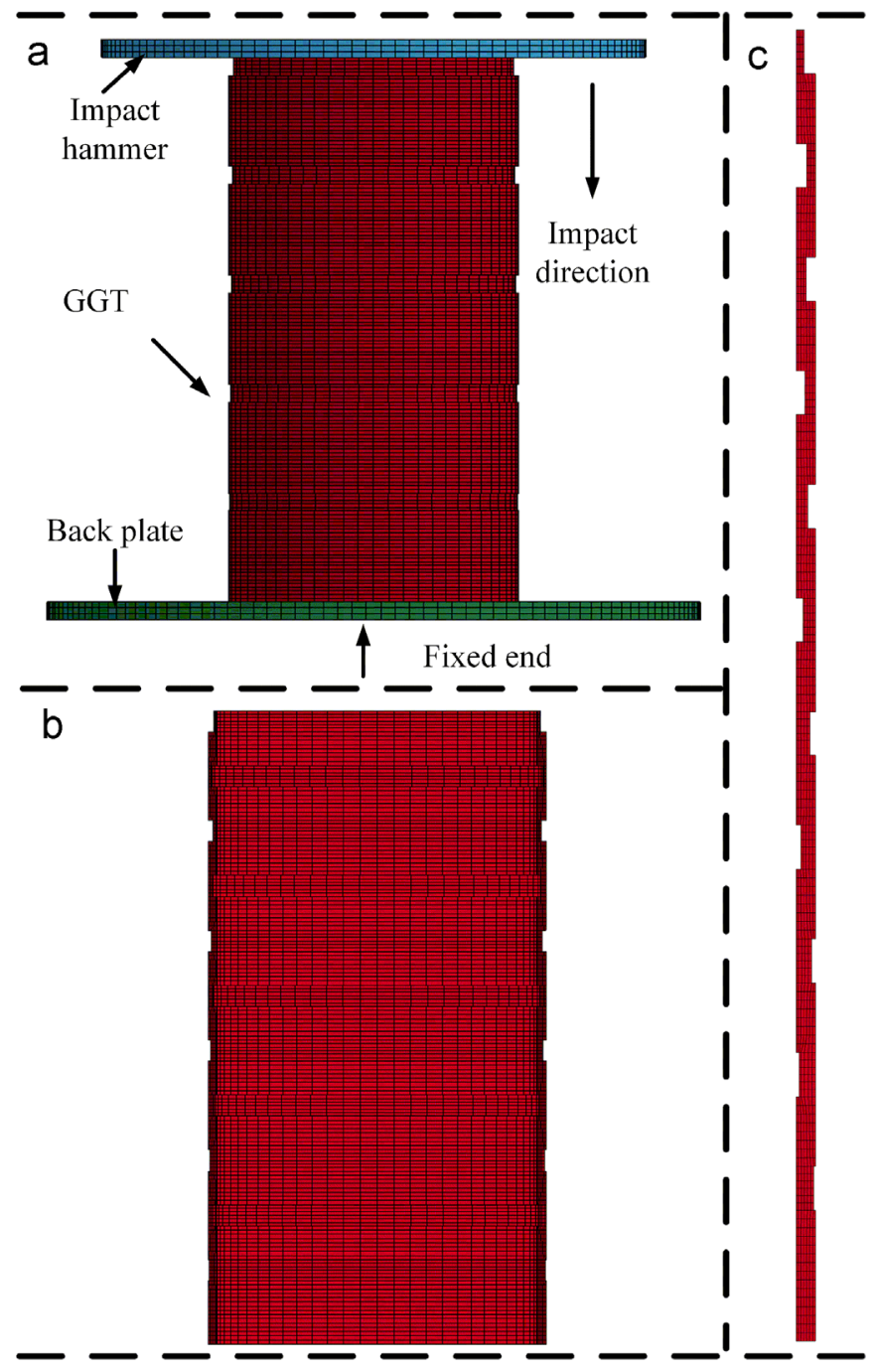

Fig. 4. Numerical model (a) parts, initial and boundary conditions of the model (b) cross-section of the GGT (c) mesh profile of the GGT tube wall.

Table 1

Material parameters for each part in the numerical model.

\begin{tabular}{llllll}
\hline Parts & Material & $\begin{array}{l}\text { Constitutive } \\
\text { model }\end{array}$ & $E(\mathrm{GPa})$ & $\rho\left(\mathrm{kg} / \mathrm{m}^{3}\right)$ & $\mu$ \\
\hline $\begin{array}{c}\text { Impact } \\
\text { body }\end{array}$ & Steel & Rigid & 210 & $2.264 \mathrm{e} 6$ & 0.3 \\
GGT & $\begin{array}{l}\text { 304 stainless } \\
\text { steel }\end{array}$ & Johnson-Cook & 210 & 7830 & 0.3 \\
Back plate & Steel & Elastic & 210 & 7800 & 0.3 \\
\hline
\end{tabular}

\subsection{Regulation of buckling modes}

The theoretical analysis of the crushing force on the GGT is deduced under the premise of standard concertina folds forming at the gradient grooves. Thus, the controllability of the plastic hinge formation is sensitive to several non-dimensional parameters including the width of groove $\left(W /\left(\mathrm{h}_{0}+h\right)\right)$, the groove depth $\left(\mathrm{h}_{0} / h\right)$ and the half wavelength $((H+W-h) / \sqrt{D h})$. In the following section, the effects of these parameters on the plastic hinge formation and integrated buckling mode are discussed.

\subsubsection{Non-dimensional width of the groove $W /\left(\mathrm{h}_{0}+h\right)$}

The fold formation assumption and theory model have been presented by many authors. Alexander [37] first presented a theoretical model to predict the average crushing force of the concertina mode, the curvature of the folds are neglected, and the plastic hinge is assumed to be a point in this model, the length of the bending curve is not included. Abramowicz and Jones [5] presented a more realistic model, they assumed that the rings bend outward and have the same curvature of opposite sense. Grzebieta' model [43] assumes that the fold has curved regions and straight regions, two curved regions are separated by a straight region and each region is one-third of the fold length. Wierzbicki et al. [40] first developed the concept of eccentric factor, the mechanism allows the rings bend inside and outside the tube midline. Singace et al. [38,39] gave out the detailed theoretical analysis and experimental validation of the eccentric factor. Both the rings in the two models above are straight and no curvature is assumed.

Based on the Alexander's model, considering the curvature of the bending groove region (and considering the eccentric factor, mentioned in Section 2), an ideal formation of a plastic hinge at a groove for GGT is that, the upper and lower rings rotate $90^{\circ}$ into a parallel state without curvature and the groove bends into a semicircle. This gives a relationship of $\mathrm{W} /\left(\mathrm{h}_{0}+h\right)=\pi / 2$ of geometry coordination which is illustrated in Fig. 7.

The effect of the non-dimensional width of the groove on the formation of the plastic hinge is numerically evaluated as a case of $\mathrm{W} /\left(\mathrm{h}_{0}+h\right)=1, \pi / 2$ and 2 . As shown in Fig. 8, two rings squeeze each other during the formation of the fold when $W /\left(h_{0}+h\right)$ is lower than $\pi / 2$. In this case, the extrusion of the rings cause additional squeezing force between two rings, which is not expected in our theory model illustrated in Section 2. Conversely, when $W /\left(h_{0}+h\right)$ is equal to or greater than $\pi / 2$, the hinges can bend smoothly without any squeezing between rings. However, a larger $W /\left(h_{0}+h\right)$ means an increased slenderness ratio of the grooves, which increases the possibility of local destabilization. Therefore, $\pi / 2$ is suggested as a reasonable value for nondimensional width of gradient grooves in all cases.

\subsubsection{Non-dimensional groove depth $h_{0} / h$}

The non-dimensional initial groove depth controls the local instability properties of the GGT. The local grooves can be regarded as uniform cylinder shells whose destabilization behavior can be predicted by non-linear large deflection theory [44]. Local buckling of the grooves themselves can be caused by overlarge $h_{0} / h$ when the critical buckling instability load of the local groove becomes lower than the whole fold formation load. When local buckling occurs, the rings between the grooves are similar with the function of ribs in reference [32], and the grooves are like minor tubes buckling between the ribs.

Fig. 9 shows numerical results of local buckling behavior of grooves at different $h_{0} / h$. The undesirable local buckling happens when the non-dimensional depth $\left(h_{0} / h \geq 0.8\right)$ and the crushing force no longer meet the value deduced from our theory. When $h_{0} /$ $h$ ranges from 0.6 to 0.8 , the groove regions bend irregularly, and the two rings cannot turn into parallel which means the buckling behavior is still out of control. In the case of $h_{0} / h \leq 0.6$, the groove regions bend smoothly to form a perfect plastic hinge which demonstrates good control effects. Therefore, since the depths of grooves in the GGT decrease gradually along the tube, we set $h_{0} /$ $h=0.6$ as the initial groove depth in all GGT configurations to ensure local controllability. It should be mentioned here that the last groove depth in all GGTs is set as $h_{\text {last }} / h=0.1$, as extremely small non-dimensional groove depth may not perpetuate the control effect. As the initial and last groove depth are determined in all GGTs, the groove number and groove depth variation $(t)$ differ with different non-dimensional half wavelengths $((H+W-h) / \sqrt{D h})$, the influence of which will be discussed in Section 5.2 .3 ahead. 


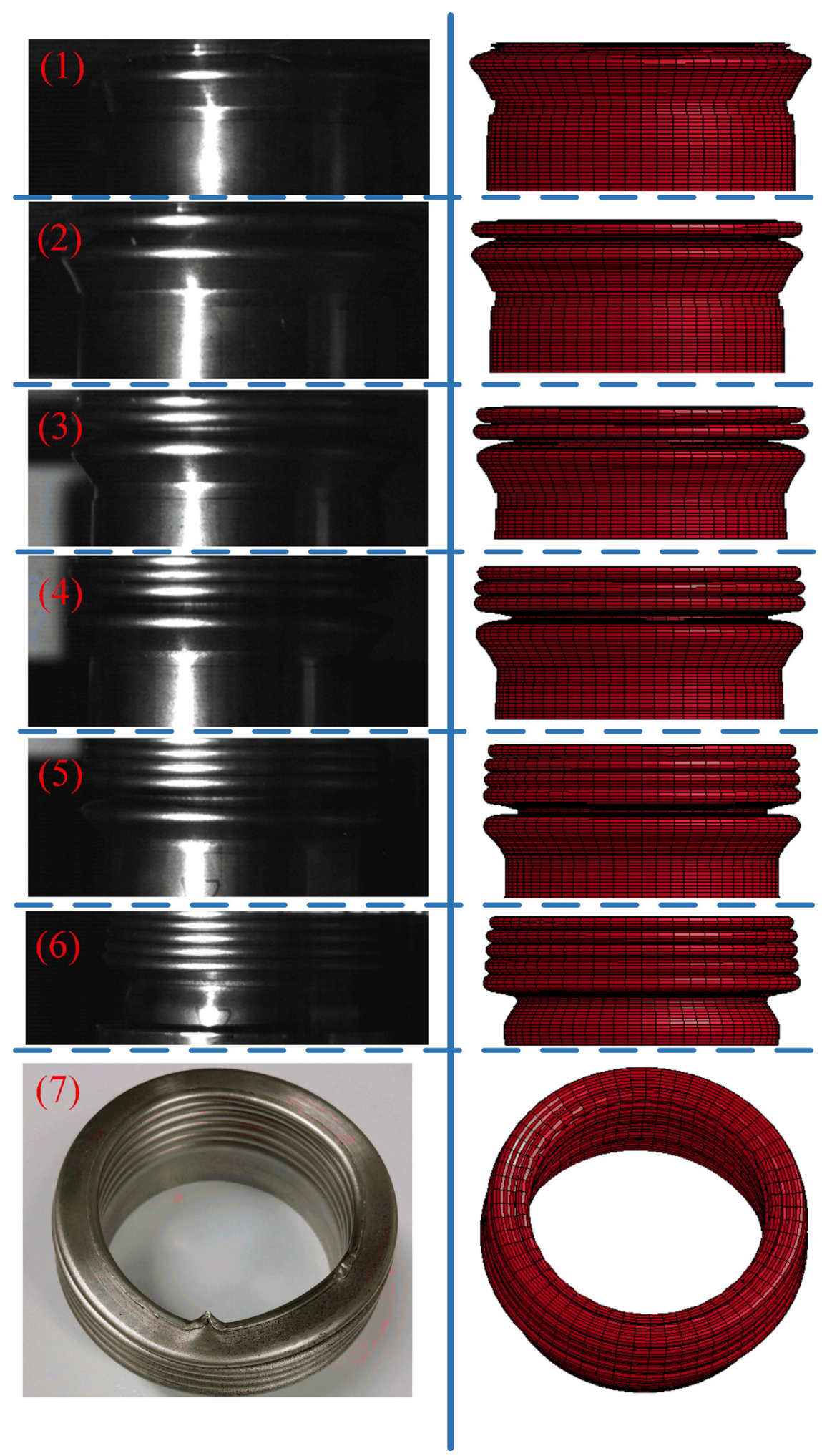

Fig. 5. Comparison of experimental and numerical results of fold formation process at $\mathrm{h}_{0} / h=0.6, \mathrm{~W} /\left(\mathrm{h}_{0}+h\right)=\pi / 2$ and $(H+W-h) / \sqrt{D h}=1$.

\subsubsection{Non-dimensional half wavelength $(H+W-h) / \sqrt{D h}$}

$\sqrt{D h}$ is widely accepted as the inherent half wavelength $[7,37,38,40]$ while $H+W-h$ is the artificial half wavelength that we hope to control during the buckling process. The ratio of $H+W-h$ to $\sqrt{D h}$ represents the competition between human and natural forces. Unfortunately, the artificially controlled concertina folds pattern can be only achieved within a certain range of parameters.
The numerical results of the integrated buckling pattern at different values of $(H+W-h) / \sqrt{D h}$ ranging from 0.7 to 1.6 are shown in Fig. 10, and the results of impact experiments at $(H+W-h) / \sqrt{D h}=1,1.2$ and 1.6 are also included. Numerical results in the case of $(H+W-h) / \sqrt{D h}=1.2$ represent a few irregularly replicated folds during the buckling process. The number of these irregular concertina folds increases in the case where $(H+W-h) / \sqrt{D h}=1.3$, and the buckling turns into diamond mode 
completely at case $(H+W-h) / \sqrt{D h}=1.4$. Two rounds of impact experiments were also carried out at $(H+W-h) / \sqrt{D h}=1.2$, one showed irregular concertina mode and the other showed diamond mode.

All of the results above demonstrate that $(H+W-h) / \sqrt{D h}=1.2$ is a critical upper limit for the artificial concertina folds. In other words, if $(H+W-h) / \sqrt{D h}$ is bigger than a critical value, the overlarge half wavelength makes the tube buckle in an uncontrollable manner. This is due to the overlarge half wavelength makes the load and energy to buckle in a concertina mode become to high, thus the diamond mode which needs lower load and energy occurs. This phenomenon and explanation fit the tube energy

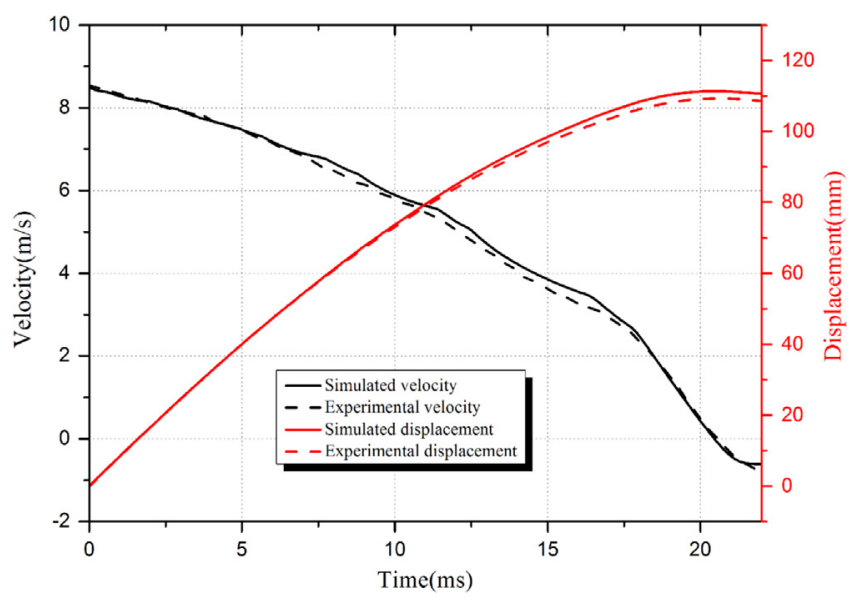

Fig. 6. Typical displacement-time and velocity-time curve from $2 \mathrm{D}$ DIC and numerical model at $\mathrm{h}_{0} / h=0.6, \mathrm{~W} /\left(\mathrm{h}_{0}+h\right)=\pi / 2$ and $(H+W-h) / \sqrt{D h}=1$.

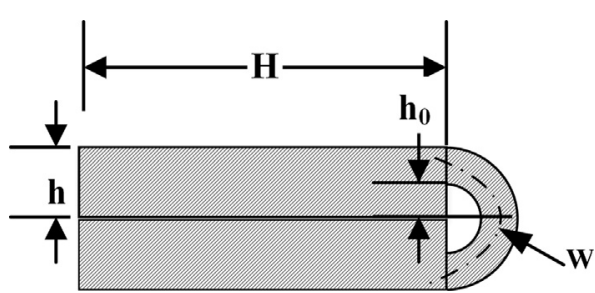

Fig. 7. Geometry relationship of perfect concertina folds at grooves. dissipation analysis by Andrews et al. [45], the concertina mode dissipates more energy than the diamond mode. Meanwhile, local tearing was observed in both numerical and experimental results in the case of $(H+W-h) / \sqrt{D h}=1.6$, which is from the local shearing behavior of the grooves induced by the irregular deformation of diamond folds. When $(H+W-h) / \sqrt{D h}<1.2$, the GGT shows good controllability and the half wavelength of the concertina folds is strictly limited to the artificial values. It should be noted that the width of the grooves could be bigger than the ring length when $(H+W-h) / \sqrt{D h}<0.7$. That has already gone beyond the concept of "defect", so the controllable non-dimensional half wavelength is determined as $0.7-1.2$ for this type of GGT.

\subsection{F-D curve of GGTs}

\subsubsection{F-D curve in controlled concertina mode}

The typical $F-D$ curve of GGT at $h_{0} / h=0.6, W /\left(h_{0}+h\right)=\pi / 2$ and $(H+W-h) / \sqrt{D h}=1$ with completely controlled concertina mode is illustrated in Fig. 11. Under the effect of the gradient grooves, the $F-D$ curve presents an upward trend with slight oscillations integrally, this upward trend can enhance the buckling stability when impacted by slender control rod. Except our

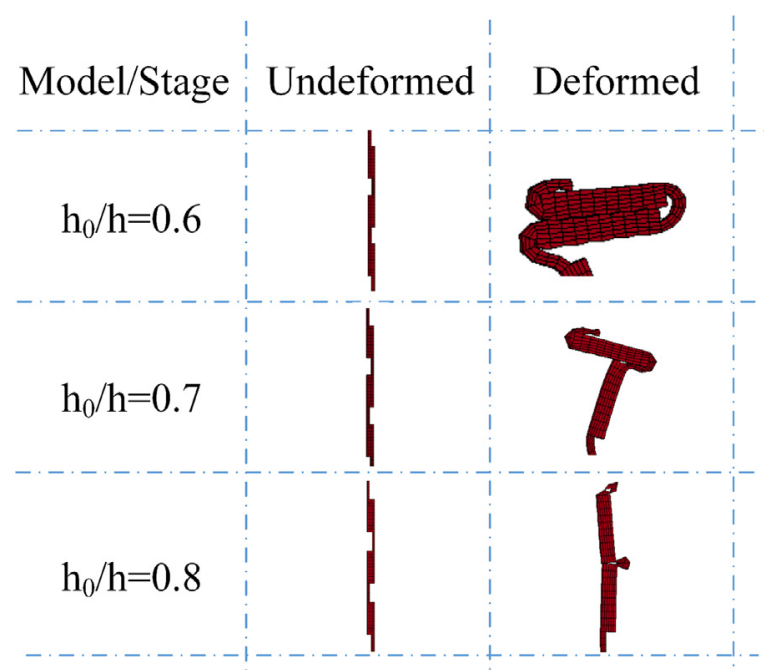

Fig. 9. Numerical deformation features of plastic hinge with different $h_{0} / h$.

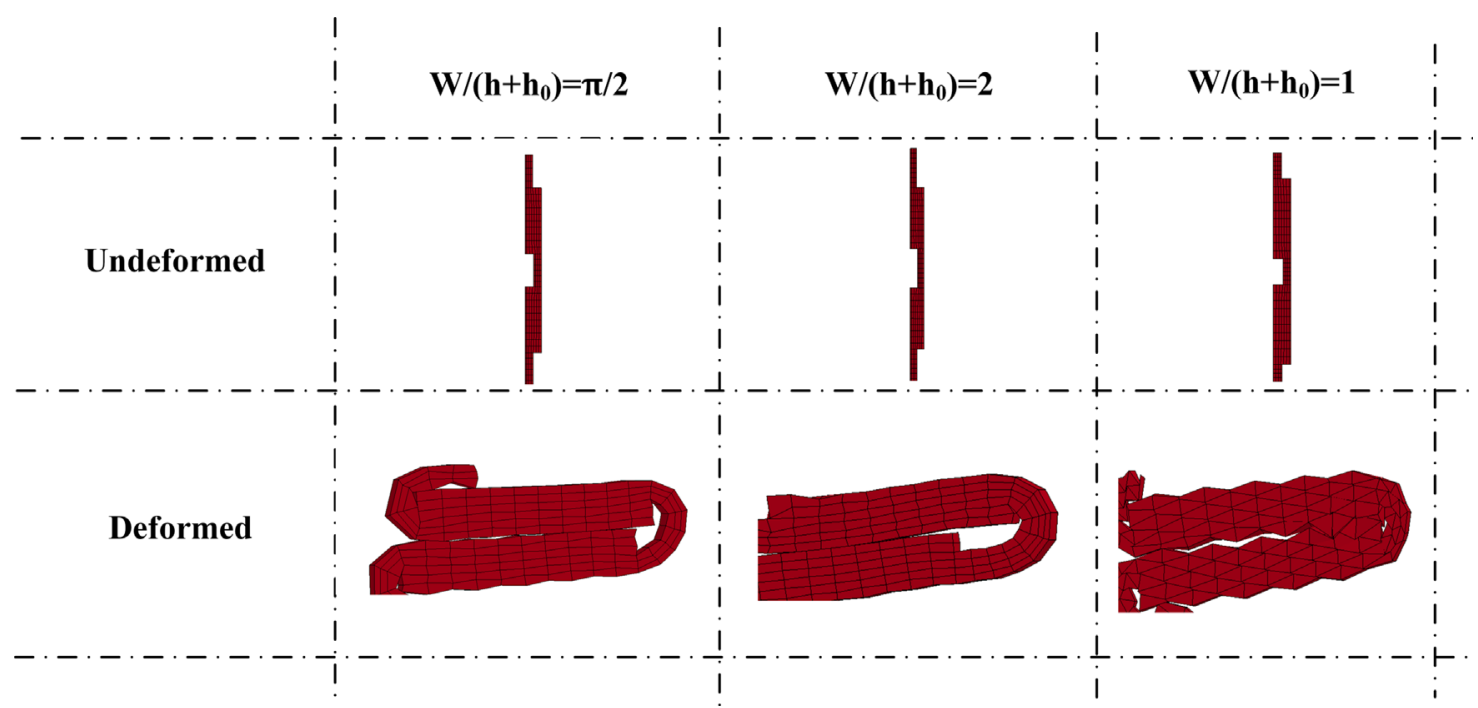

Fig. 8. Numerical deformation features of plastic hinge with different $W /\left(h_{0}+h\right)$. 


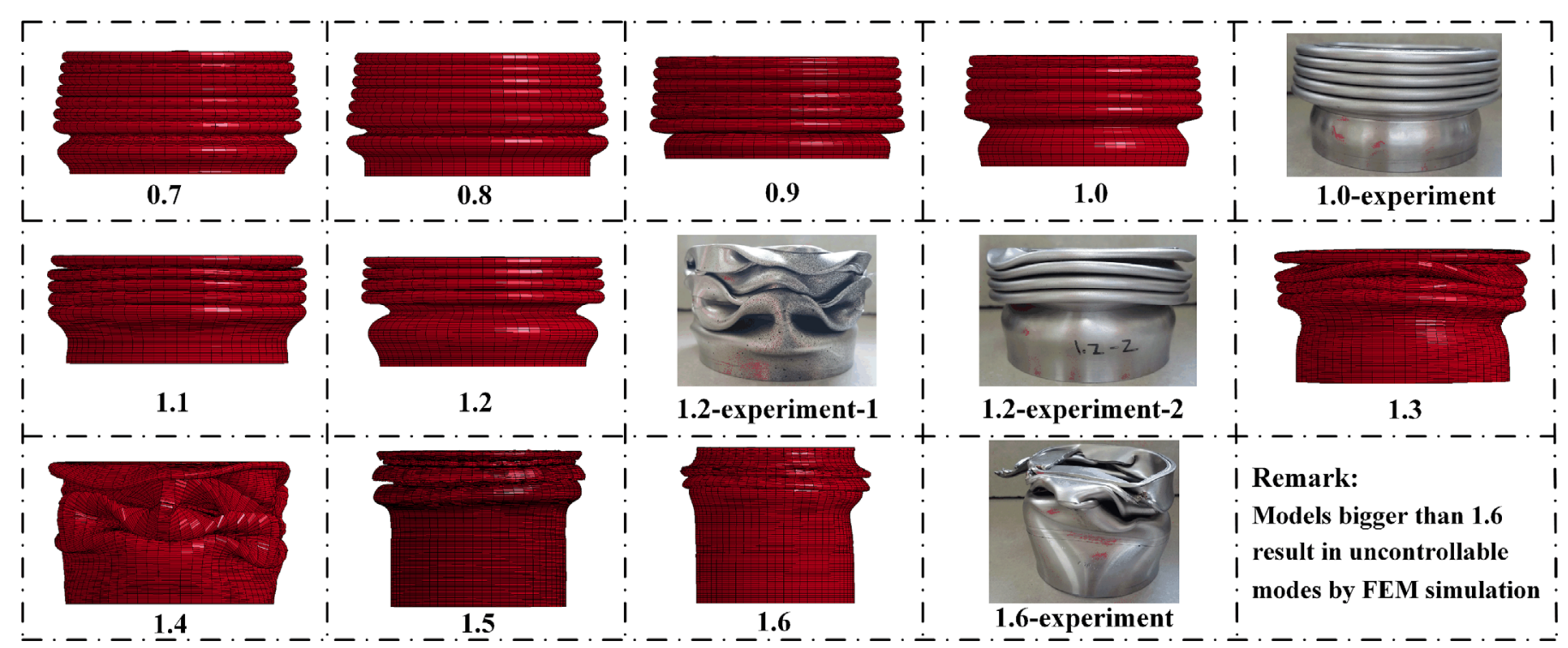

Fig. 10. Numerical and experimental results of integrated buckling patterns at different $(H+W-h) / \sqrt{D h}$ ranging from 0.7 to 1.6 .

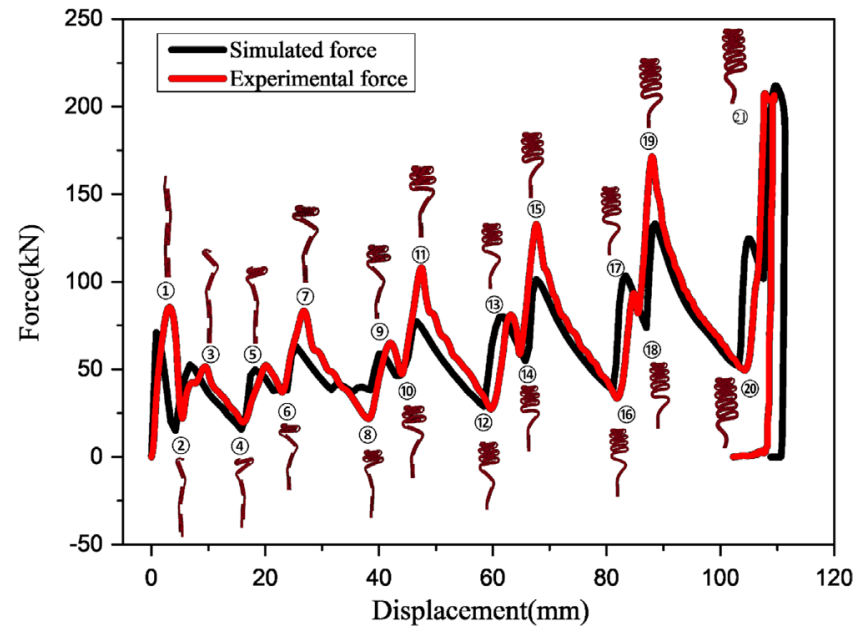

Fig. 11. Typical force-displacement curve of GGT.

gradient grooves arrangement method, the upward trend can also be achieved by tube diameter variation [35], tube wall thickness and diameter variation simultaneously [36], gradient foam filling together with tube wall thickness variation [46]. The double peak feature of each fold formation is because of the eccentric effect; the same feature has been observed and studied by Singace et al. $[38,39]$. As the eccentric factor of this model is about 0.6 , the crushing force inward on the plastic hinge will be lower than the outward, which arises the discrepancy of two peak values corresponding to the initial bending inward and outward respectively.

\subsubsection{Comparison of the theoretical, experimental and numerical} results

In order for a comparison to be made with the theoretical results, the numerical and experimental $F-D$ curves have been sectionalized into $h_{\text {mid }}$ curves of average crushing force (sectionalized $F-D$ curves). As shown in Fig. 12, the crushing displacement is represented by the non-dimensional middle groove wall thickness of the $n$th fold $\left(h_{\text {mid }}\right)$ and the transient crushing force is represented by the sectionalized average crushing force derived from the ratio of energy absorption and the crushing displacement through the $n$th fold.

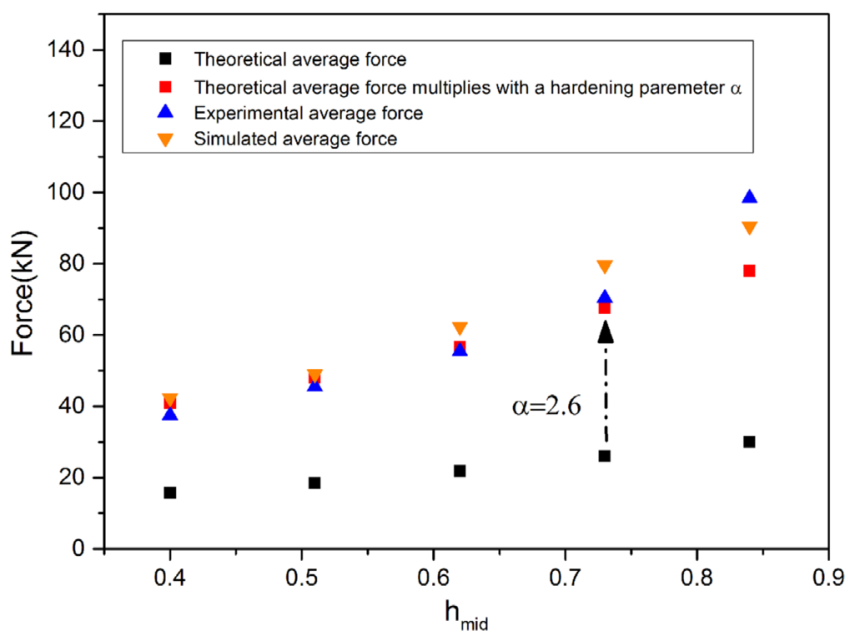

Fig. 12. Comparison of theoretical, numerical and experimental results of sectionalized $F-D$ curve at $h_{0} / h=0.6, W /\left(h_{0}+h\right)=\pi / 2$ and $(H+W-h) / \sqrt{D h}$.

The sectionalized $F-D$ curves represent an obvious upward trend. The theoretical results can predict this trend even though the exact values are lower than the experimental and numerical results. The discrepancy is mainly derived from a consideration of strain hardening and strain rate hardening. Generally, the quasistatic theory can also be applied in low-velocity impact conditions when the strain and strain rate hardening effect are considered by multiplying a hardening factor [29] by the static crushing force as expressed in Eq. (18). This hardening parameter $(\alpha)$ is determined as 2.6 for our configurations and impact conditions, thus demonstrating good agreement among the theoretical, experimental and numerical results.

$F_{n}^{\text {impact }}=\alpha F_{n}^{\text {static }}$

Our theory predicts a quadratic upward trend of the sectionalized crushing force on the GGT. This upward trend is sensitive to the non-dimensional half wavelengths $((H+W-h) / \sqrt{D h})$ as shown in Fig. 13. Within a controllable range, the upward trend is gradually weakened with the increasing $(H+W-h) / \sqrt{D h}$, the numerical sectionalized $F-D$ curves fit well with the theoretical curves. The upward trend influencing by the non-dimensional half wavelength $(H+W-h) / \sqrt{D h}$ provides further evidence for the controllability of the GGT. 


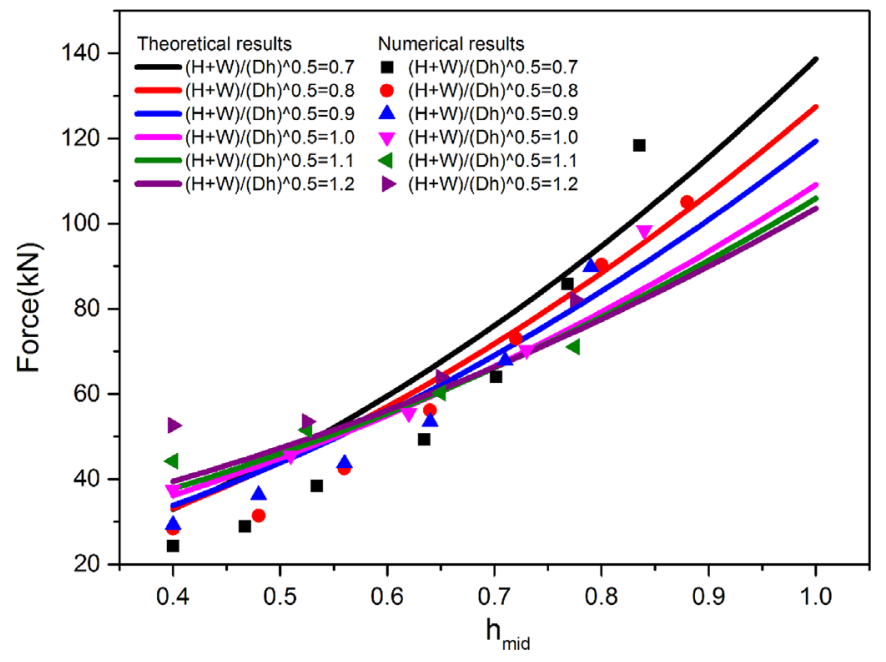

Fig. 13. Numerical sectionalized $F-D$ curves of GGTs with different $(H+W-h) / \sqrt{D h}$.

Table 2

Coefficients of the theoretical force formulation for different $(H+W-h) / \sqrt{D h}$.

\begin{tabular}{llll}
\hline$(H+W-h) / \sqrt{D h}$ & $\begin{array}{l}\text { Quadratic } \\
\text { coefficient }\end{array}$ & $\begin{array}{l}\text { Primary } \\
\text { coefficient }\end{array}$ & $\begin{array}{l}\text { Constant } \\
\text { coefficient }\end{array}$ \\
\hline 0.7 & 108.4 & 24.3 & 6.0 \\
0.8 & 94.8 & 24.5 & 8.2 \\
0.9 & 84.3 & 24.7 & 10.5 \\
1 & 69.0 & 25.0 & 15.2 \\
1.1 & 63.2 & 25.1 & 17.6 \\
1.2 & 58.3 & 25.1 & 20.1 \\
\hline
\end{tabular}

\subsubsection{Theoretical analysis of sectionalized $F-D$ curves}

The quadratic term, the primary term and the constant term of the theoretical sectionalized average force can be obtained from formulation (16) and (18) for different $(H+W-h) / \sqrt{D h}$ as shown in Table 2. It can be seen from formulations (15) and (16) that the contribution of the quadratic term $\alpha \pi^{2} Y D h^{2} h_{\text {mid }}^{2} / 2 \sqrt{3}(H+W-h)$ of crushing force is mainly derived from plastic bending in local gradient grooves. The bending force increases rapidly at a speed that is proportional to $h_{\text {mid }}^{2}$. It is demonstrated that the quadratic coefficient $\alpha \pi^{2} Y D h^{2} / 2 \sqrt{3}(H+W-h)$ is sensitive to the nondimensional half wavelength $(H+W-h) / \sqrt{D h}$. As shown in Fig. 14a, the upward trend of crushing force is obviously suppressed at bigger $(H+W-h) / \sqrt{D h}$. As we know, since the bending state of a complete concertina fold is almost the same, such as the concertina models discussed in Section 5.2.1 [7,37,38,40] and the groove bending model we assumed in Fig. 7 , the bending force is mainly dependent on the thickness of the grooves that participate in bending. For the same $h_{\text {mid }}$, the energy absorbed by bending is the same, thus bigger $(H+W-h) / \sqrt{D h}$ reduces the energy absorption efficiency as the crushing displacement increasing for a fold, and this causes the upward trend of bending force to level off.

The primary term $\alpha \pi Y h\left(W^{2}+2 H W\right) h_{\text {mid }} / 2(H+W-h)$ represents the contribution of stretching and compressing of the grooves. The crushing force from this part is linearly increased during the buckling process as shown in Fig. 14b. The primary coefficient $\alpha \pi Y h\left(W^{2}+2 H W\right) / 2(H+W-h)$ is insensitive to the non-dimensional half wavelength $(H+W-h) / \sqrt{D h}$. This insensitivity is mainly derived from that: for bigger half wavelength, although the crushing displacement for a fold increased, the amount of stretching and compressing deformation will be increased too, which led to a neutralizing effect. a

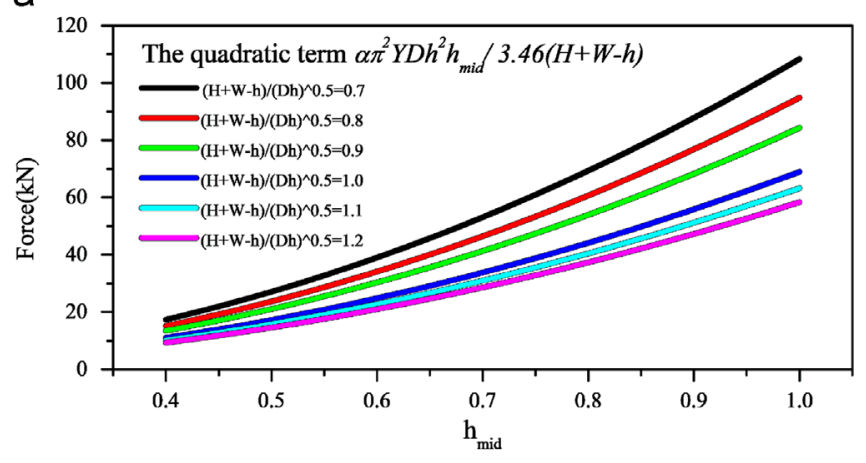

b

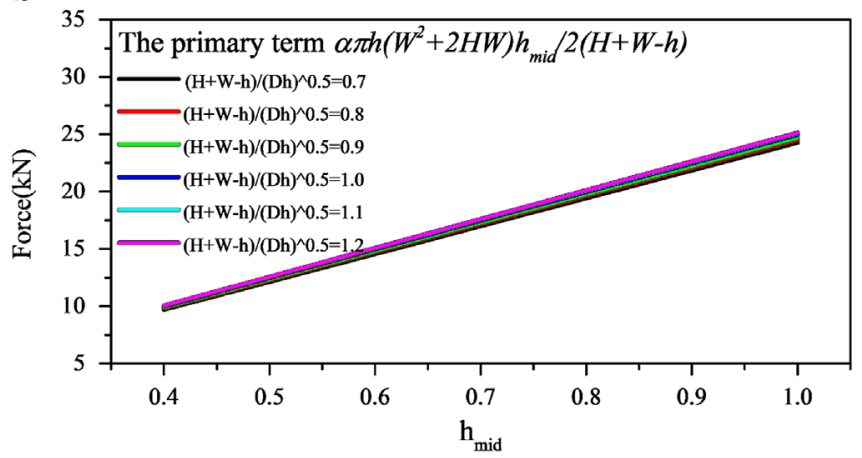

C

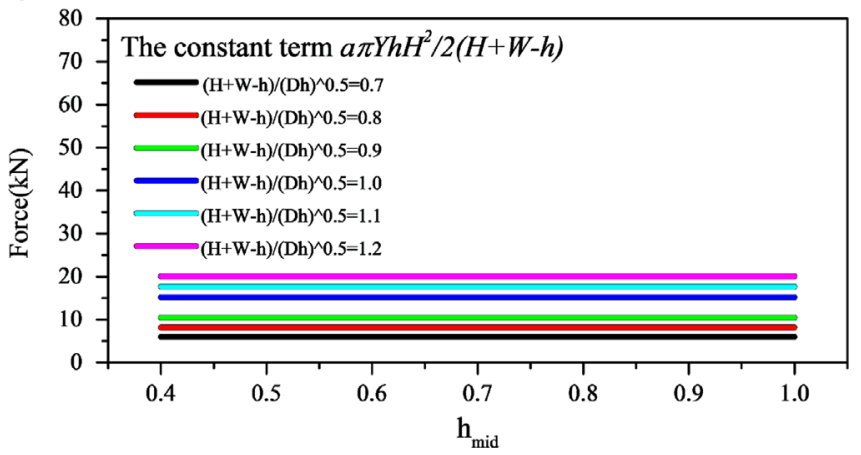

Fig. 14. The three terms of the theoretical force with different $(H+W-h) / \sqrt{D h}$ (a) the quadratic term; (b) the primary term; (c) the constant term.

The contribution of constant term $\alpha \pi Y h H^{2} / 2(H+W-h)$ is derived from stretching and compressing of the rings between three grooves. Since the identical half wavelength is set in a unique GGT, the crushing force from this part is independent of $h_{\text {mid }}$ as shown in Fig. 14c. However, since the amount of stretching and compressing deformation increases at greater half wavelength, a larger crushing force will be output for this part due to more material participation in energy absorption, even though the crushing displacement increases. This is confirmed by the positive correlation with $(H+W-h) / \sqrt{D h}$ as illustrated in Table 2 .

We can see in Fig. 14 that the quadratic term contributes the most energy absorption for the entire process, the primary term contributes the second most, and the constant term makes the least energy absorption contribution. However, as the quadratic and constant terms have negative and positive correlations with $(H+W-h) / \sqrt{D h}$, the gap between the constant terms and other two terms for bigger $(H+W-h) / \sqrt{D h}$ becomes smaller.

\subsection{Application of GGT in HTR}

The proactive regulation effects of GGT is examined by free-fall impact experiment in control rod channel, with the same experiment 

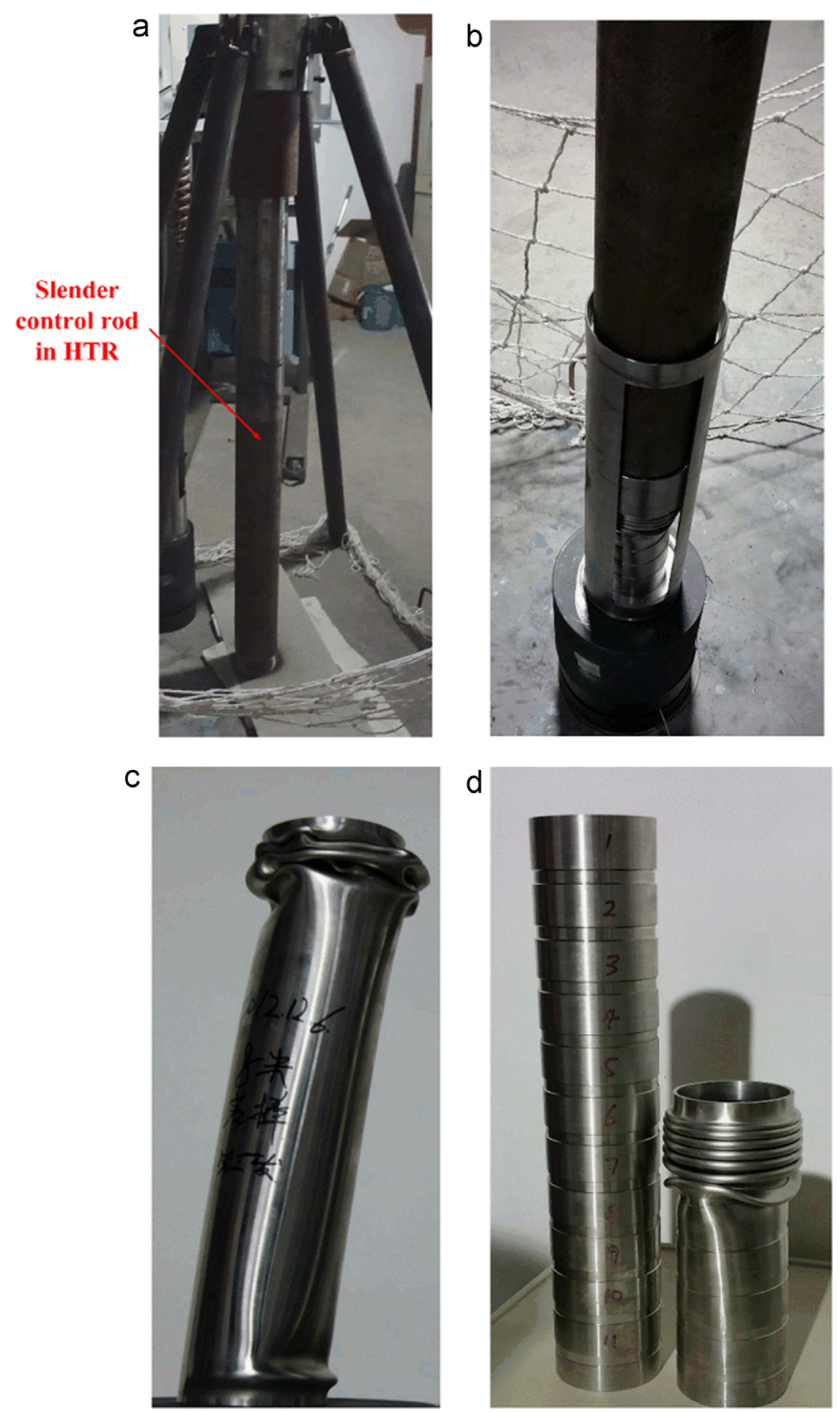

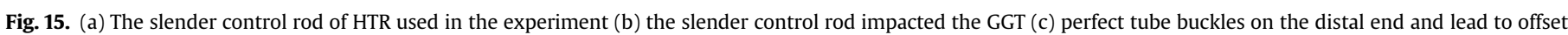
laterally; (d) GGT buckles in controlled concertina mode.

setup in reference [27]. Fig. 15 shows the slender control rod and crushed perfect tube and GGT. Perfect tubes have tendencies to buckle from distal end, resulting laterally offset from the impact axis. In contrast, the GGT exhibits good stability with standard concertina folds and consistent buckling sequences, and no lateral offset happens in all freefall impact experiments.

\section{Conclusions}

In this paper, a method to proactively regulate the buckling process of a thin-walled tube has been explored using the gradient defect. The gradient grooved tube (GGT) is designed to protect graphite in high temperature gas-cooled reactor (HTR) from the free-fall impact of slender control rod. A sectionalized theory for crushing force of the GGT is developed and predicts a quadratic upward trend which is also confirmed by experimental and simulated results. Several non-dimensional parameters having significant influences on crushing force and buckling modes are studied comprehensively using theoretical, experimental and numerical methods. The slender control rod free-fall experiments have been performed to the perfect tube and GGT respectively. The main findings of this paper are as follows: 
1. The non-dimensional groove width $\left(W /\left(\mathrm{h}+\mathrm{h}_{0}\right)\right)$ is determined as $\pi / 2$ based on the geometric coordination of plastic hinge formation. The non-dimensional groove depth $h_{0} / h=0.6$ is determined as an upper limit value for regular bending which is the premise of proactive regulation of the buckling of the GGT. The non-dimensional half wavelength $(H+W-h) / \sqrt{D h}$ has a great effect on the integral buckling mode of the GGT. This upper limit value for controllable concertina mode is determined as $(H+W-h) / \sqrt{D h}=1.2$ by both experiments and simulations.

2. The integral and sectionalized $F-D$ curves of the GGT show a quadratic upward trend to realize a more stable crushing process. This upward trend shows a negative correlation with the non-dimensional half wavelength $(H+W-h) / \sqrt{D h}$, showing controlablle by this parameter within a certain range.

3. The relationship between the three terms of the theoretical quadratic crushing force and $(H+W-h) / \sqrt{D h}$ has been analyzed theoretically. The quadratic term is mainly derived from the plastic bending in local gradient grooves, can be obviously suppressed with bigger $(H+W-h) / \sqrt{D h}$. The primary term is mainly derived from the stretching and compressing of the grooves, showing insensitive to $(H+W-h) / \sqrt{D h}$. The constant term of crushing force is mainly derived from the stretching and compressing of the rings between three grooves, having a positive correlation with $(H+W-h) / \sqrt{D h}$.

4. Finally, the free-fall impact experiments demonstrate that GGT has a better stability than perfect tube when used as an absorber to protect the graphite in HTR from slender control rod.

\section{Acknowledgments}

The authors would like to thank the National Natural Science Foundation of China (Grant no. 11502274, 11002150, 11332011, 11402277) and the Basic Research Equipment Project of the Chinese Academy of Sciences (YZ200930) for financial support.

\section{References}

[1] Olabi AG, Morris E, Hashmi MSJ. Metallic tube type energy absorbers: a synopsis. Thin-Walled Struct 2007;45:706-26.

[2] Alghamdi AAA. Collapsible impact energy absorbers: an overview. ThinWalled Struct 2001:29:189-213.

[3] Lu G, Yu T. Energy absorption of structures and materials. Elsevier; 2003.

[4] Jones N. Structural impact. Cambridge University Press; 2011.

[5] Abramowicz W, Jones N. Dynamic progressive buckling of circular and square tubes. Int J Impact Eng 1986:4:243-70.

[6] Gupta NK, Velmurugan R. An analysis of axi-symmetric axial collapse of round tubes. Thin-Walled Struct 1995;22:261-74.

[7] Bardi FC, Yun HD, Kyriakides S. On the axisymmetric progressive crushing of circular tubes under axial compression. Int J Solids Struct 2003;40:3137-55.

[8] Zhang X, Zhang H. Energy absorption of multi-cell stub columns under axial compression. Thin-Walled Struct 2013;68:156-63.

[9] Al-Qureshi H. Mechanics of static and dynamic inversion processes. Int J Mech Sci 1997;39:147-61.

[10] Reid S, Harrigan J. Transient effects in the quasi-static and dynamic internal inversion and nosing of metal tubes. Int J Mech Sci 1998:40:263-80.

[11] Qiu X, He L, Gu J, Yu X. A three-dimensional model of circular tube under quasi-static external free inversion. Int J Mech Sci 2013;75:87-93.

[12] Yu X, Qiu X, Yu TX. Analysis of the free external inversion of circular tubes based on deformation theory. Int J Mech Sci 2015;100:262-8.

[13] Reddy TY, Reid SR. Axial splitting of circular metal tubes. Int J Mech Sci 1986;28:111-31.
[14] Huang X, Lu G, Yu TX. On the axial splitting and curling of circular metal tubes. Int J Mech Sci 2002;44:2369-91.

[15] Jiang P, Wang W, Zhang GJ. Size effects in the axial tearing of circular tubes during quasi-static and impact loadings. Int J Impact Eng 2006;32:2048-65.

[16] DeRuntz JA, Hodge P. Crushing of a tube between rigid plates. J Appl Mech 1963;30:391-5.

[17] Gupta NK, Sekhon GS, Gupta PK. Study of lateral compression of round metallic tubes. Thin-Walled Struct 2005;43:895-922.

[18] Reddy TY, Reid S. Lateral compression of tubes and tube-systems with side constraints. Int J Mech Sci 1979;21:187-99.

[19] Baroutaji A, Gilchrist M, Smyth D, Olabi A. Crush analysis and multi-objective optimization design for circular tube under quasi-static lateral loading. ThinWalled Struct 2015;86:121-31.

[20] Baroutaji A, Gilchrist MD, Olabi AG. Quasi-static, impact and energy absorption of internally nested tubes subjected to lateral loading. Thin-Walled Struct 2016:98:337-50.

[21] Baroutaji A, Morris E, Olabi AG. Quasi-static response and multi-objective crashworthiness optimization of oblong tube under lateral loading. ThinWalled Struct 2014;82:262-77.

[22] Reid S, Bell W. Influence of strain hardening on the deformation of thin rings subjected to opposed concentrated loads. Int J Solids Struct 1982;18:643-58.

[23] Lu G. A study of the crushing of tubes by two indenters. Int J Mech Sc 1993:35:267-78.

[24] Liu K, Zhao K, Gao Z, Yu T. Dynamic behavior of ring systems subjected to pulse loading. Int J Impact Eng 2005;31:1209-22.

[25] Yuanqiang W, Xingzhong D, Huizhong Z, Zhiyong H. Design and tests for the HTR-10 control rod system. Nucl Eng Des 2002;218:147-54.

[26] Takamatsu M, Sekine T, Aoyama T, Uchida M, Kotake S. Demonstration of control rod holding stability of the self actuated shutdown system in Joyo for enhancement of fast reactor inherent safety. J Nucl Sci Technol 2007:44:511-7.

[27] Yang Z, Yan H, Huang C, Diao X, Wu X, Wang S, Lu L, Liao L, Wei Y. Experimental and numerical study of circular, stainless thin tube energy absorber under axial impact by a control rod. Thin-Walled Struct 2014;82:24-32.

[28] Guillow SR, Lu G, Grzebieta RH. Quasi-static axial compression of thin-walled circular aluminium tubes. Int J Mech Sci 2001;43:2103-23.

[29] Abramowicz W, Jones N. Dynamic axial crushing of circular tubes. Int J Impac Eng 1984;2:263-81.

[30] Yuen SCK, Nurick GN. The energy-absorbing characteristics of tubular structures with geometric and material modifications: an overview. Appl Mech Rev 2008;61:020802.

[31] Thornton PH, Magee CL. Interplay of geometric and materials variables in energy-absorption. J Eng Mater Technol Trans ASME 1977;99:114-20.

[32] Adachi T, Tomiyama A, Araki W, Yamaji A. Energy absorption of a thin-walled cylinder with ribs subjected to axial impact. Int J Impact Eng 2008;35:65-79.

[33] Daneshi GH, Hosseinipour SJ. Grooves effect on crashworthiness characteristics of thin-walled tubes under axial compression. Mater Des 2002;23:611-7.

[34] Hosseinipour SJ, Daneshi GH. Energy absorbtion and mean crushing load of thin-walled grooved tubes under axial compression. Thin-Walled Struct $2003 ; 41: 31-46$

[35] Gupta NK, Sheriff NM, Velmurugan R. A study on buckling of thin conica frusta under axial loads. Thin-Walled Struct 2006;44:986-96.

[36] Zhang X, Zhang H, Wen Z. Axial crushing of tapered circular tubes with graded thickness. Int J Mech Sci 2015;92:12-23.

[37] ALEXANDER JM. An approximate analysis of the collapse of thin cylindrical shells under axial loading. O J Mech Appl Math 1960;13:10-5.

[38] Singace AA, Elsobky H, Reddy TY. On the eccentricity factor in the progressive crushing of tubes. Int J Solids Struct 1995;32:3589-602.

[39] Singace AA, Elsobky H. Further experimental investigation on the eccentricity factor in the progressive crushing of tubes. Int J Solids Struct 1996:33:3517-38.

[40] Wierzbicki T, Bhat SU, Abramowicz W, Brodkin D. Alexander revisited-a two folding elements model of progressive crushing of tubes. Int J Solid Struc 1992:29:3269-88.

[41] Johnson GR, Cook WH. Fracture characteristics of three metals subjected to various strains, strain rates, temperatures and pressures. Eng Fract Mech $1985 ; 21: 31-48$

[42] Wei Y. Experimental research on welding technique and mechanic properties of dissimilar stainless steel laser welding. China: Institute of Mechanics, Chinese Academy of Sciences; 2010.

[43] Grzebieta RH. An alternative method for determining the behaviour of round stocky tubes subjected to an axial crush load. Thin-Walled Struct 1990;9:61-89.

[44] Karman TV, Tsien H-S. The buckling of thin cylindrical shells under axial compression. J Spacecr Rocket 2003;40:898-907.

[45] Andrews KRF, England GL, Ghani E. Classification of the axial collapse of cylindrical tubes under quasi-static loading. Int J Mech Sci 1983;25:687-96.

[46] zhang Y, Lu M, Sun G, Li G, Li Q. On functionally graded composite structures for crashworthiness. Compos Struct 2015;132:393-405. 